EPJ manuscript No.

(will be inserted by the editor)

\title{
Fracture and Friction: Stick-Slip Motion
}

\author{
Efim A. Brener ${ }^{1}$, S. V. Malinin ${ }^{1,2 a}$, and V. I. Marchenko ${ }^{3}$ \\ ${ }^{1}$ Institut für Festkörperforschung, Forschungszentrum Jülich \\ ' 2 L.D. Landau Institute for Theoretical Physics, RAS, 119334, Kosygin str. 2, Moscow, Russia \\ ${ }_{3}^{3}$ P.L. Kapitza Institute for Physical Problems, RAS, 119334, Kosygin str. 2, Moscow, Russia
}

September 24, 2018

\begin{abstract}
We discuss the stick-slip motion of an elastic block sliding along a rigid substrate. We argue that for a given external shear stress this system shows a discontinuous nonequilibrium transition from a uniform stick state to uniform sliding at some critical stress which is nothing but the Griffith threshold for crack propagation. An inhomogeneous mode of sliding occurs, when the driving velocity is prescribed instead of the external stress. A transition to homogeneous sliding occurs at a critical velocity, which is related to the critical stress. We solve the elastic problem for a steady-state motion of a periodic stick-slip pattern and derive equations of motion for the tip and resticking end of the slip pulses. In the slip regions we use the linear viscous friction law and do not assume any intrinsic instabilities even at small sliding velocities. We find that, as in many other pattern forming system, the steady-state analysis itself does not select uniquely all the internal parameters of the pattern, especially the primary wavelength. Using some plausible analogy to first order phase transitions we discuss a "soft" selection mechanism. This allows to estimate internal parameters such as crack velocities, primary wavelength and relative fraction of the slip phase as function of the driving velocity. The relevance of our results to recent experiments is discussed.
\end{abstract}

PACS. 62.20.Mk Fatigue, brittleness, fracture, and cracks - 46.50.+a Fracture mechanics, fatigue and cracks $-46.55 .+\mathrm{d}$ Tribology and mechanical contacts - 62.20.Qp Tribology and hardness

\section{Introduction}

The nature of sliding friction and especially of inhomogeneous modes of sliding is a fundamental physical problem of prime practical importance [1. Studying the friction of rubber along a smooth glass substrate, Schallamach [2] observed an inhomogeneous mode of sliding. Under some conditions homogeneous sliding becomes unstable and a quasiperiodic pattern of detached zones is formed. Recent experimental observations of Rubio and Galeano [3], 'Baumberger, Caroli, and Ronsin [4]5], on the frictional motion of sheared gels sliding along a glass surface also indicate the existence of self-healing pulses and inhomogeneous modes of sliding. A regime of periodic stick-slip has been observed in a limited range of small shear rates 4, 5. It bifurcates towards stationary sliding at some critical driving velocity. The slip pulses traverse the sample with a velocity much larger than the driving velocity but still much smaller than the speed of sound. The importance of the slow crack-like fronts for the onset of the frictional slip was also stressed in [6].

Slip pulses in gels seem to be very different from Schallamach waves and "brittle" pulses studied by Gerde and Marder [7] since no observable opening occurs at the in-

\footnotetext{
a Present address: Institut für Theoretische Physik, Universität zu Köln, 50937 Köln, Germany
}

terface. In this respect, they are more comparable to selfhealing cracks, suggested by Heaton 8 in the context of seismic events.

Stick-slip motion with the transition to sliding above some critical velocity was also observed in studies of friction in an ultrathin layer of lubricant between two atomically flat surfaces [9. This behavior was attributed to the confinement-induced freezing of the lubricant and its melting due to shear stress.

Recent investigations (see, for example, 10] and references therein) point towards an essential importance of the underlying friction law in the slip state. It has been proved that the simple Coulomb friction leads to the so-called "illposedness" of the linear stability problem for small inhomogeneous perturbations of the stress and strain fields in a sliding mode [10. Moreover, Caroli 11] has shown that the existence of slow, periodic slip pulses is incompatible with the Coulomb friction law.

In our previous paper [12] we developed a conceptually simple model which is compatible with an inhomogeneous mode of sliding, the existence of slowly moving slip pulses and the "shear melting" phenomenon. We discussed the propagation of a shear crack (Mode II crack) along the interface between two dissimilar materials. The crack edge separates two states of the interface, "stick" and "slip". We assumed that the interface is flat with a strong ad- 
hesion contact. In the presence of roughness, the assumption of strong adhesion and full contact at the interface is presumably only reasonable for "soft" materials with a relatively small shear modulus. Gels are clearly materials of this type.

In the slip region we assumed a simple linear viscous friction law, namely, that the shear stress is proportional to the sliding velocity. This, from the theoretical point of view strongly motivated law, is usually not discussed in the literature since it does not lead to the so-called static friction phenomenon observed experimentally. However, in our description static friction appears in a natural way as the usual Griffith threshold for crack propagation. The important point is that, before the system goes into a sliding mode, a shear crack should traverse the sample. This requires a finite shear stress since the stick state of the interface is energetically more favorable.

We note that the above mentioned interface properties in our model are fully coupled to the bulk elasticity of a gel leading to a rich spectrum of phenomena where two intriguing problems, crack propagation and interface friction, come together.

In the present paper we attempt to find a solution of this model which represents a periodic pattern of stick-slip motion. The paper is organized as follows:

In the next section we formulate the problem of the periodic pattern which appears in an elastic solid block sliding on a flat rigid substrate (see Fig. 1). We formulate the boundary conditions for this elastic problem and introduce the thermodynamics of two states of the interface: stick and slip. Each slip pulse can be considered as a mode II crack which is bounded by two crack edges: the pulse tip and the resticking end. In order to formulate equations of motion for such cracks we have to solve the elastic problem and calculate the energy flux into the crack edges.

In section [3] we solve the elastic problem in two limiting cases: the height of the block is much larger than the lateral scale of the pattern and the opposite limit. This section is rather technical but eventually provides expressions for the energy flux into the crack tips. It turns out that, as in many pattern forming systems (for example, directional solidification or eutectic growth [13]), the steady-state description itself does not allow to select the primary wavelength of the pattern. We will see that the degeneracy of our system is even higher than in crystal growth problems.

In section 4 we give some plausible arguments based on the analogy to the first order phase transitions which lead to the "soft" selection of the periodic pattern. We predict a regime of stick-slip motion in a limited range of small shearing rates and a transition towards stationary sliding at some critical driving velocity. In section [5] we discuss our results and their possible relevance to existing experimental observations. Technical details are presented in the Appendix.

\section{Formulation of the problem}

Consider an elastic solid sliding on a flat rigid substrate. Assume that the elastic solid occupies the space $0<y<$ $H$, and let $(x, y, z)$ be a coordinate system with the plane $y=0$ corresponding to the surface of the solid, see Fig. 1 We discuss the plane strain situation with $u_{z}=0$, where

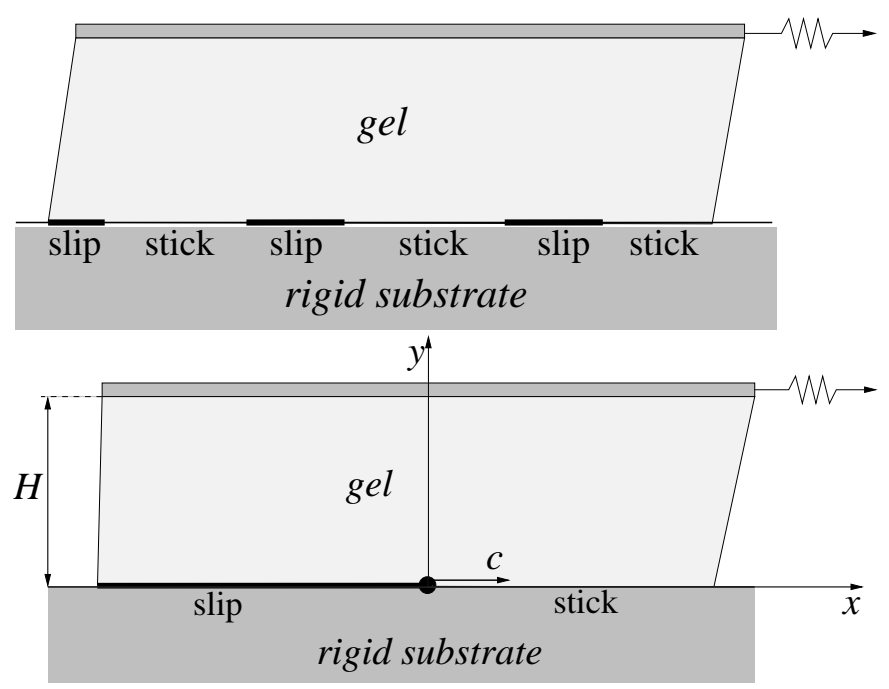

Fig. 1. (top) an elastic body sliding on a rigid substrate; (bottom) motion of a single crack tip.

$\mathbf{u}$ is the displacement vector and static elasticity since all motions are expected to be slow compared to the sound velocities.

Let us discuss the boundary conditions. First of all we assume that displacements at the top boundary of the block are the same as for the top of the rigid plate: $u_{y}=$ 0 and $u_{x}=v t+$ const are uniform for $y=H$. Here $v$ is the driving velocity of the upper rigid plate relative to the bottom rigid substrate; $t$ is time. We assume also that the bottom interface can be in two states: "stick" and "slip". The boundaries between these two states are described by the crack edges which move with a velocity $c$ in the $x$ direction. In the stick regions $u_{y}=0$ and the sliding velocity also vanishes, $\dot{u}_{x}=0$ for $y=0$. In the stationary situation, when all stick-slip boundaries drift with the same velocity $c$, displacement derivatives depend on $x$ and time $t$ only in the combination $x-c t$. Therefore, in the stick regions there is a constant deformation $u_{x x}=$ $V_{0} \equiv v / c$.

In the slip regions we assume that the two solids are always in contact, $u_{y}=0$ for $y=0$, while we allow for a finite relative sliding velocity $\dot{u}_{x}$. This sliding velocity leads to frictional shear stress at the interface, $\sigma_{x y}=\sigma_{x y}\left\{\dot{u}_{x}(t, x)\right\}$. In the following, a simple linear viscous friction law will be considered, $\sigma_{x y}=\beta \dot{u}_{x}$, with $\beta$ being the friction coefficient.

Let us turn now to thermodynamical aspects of the problem Since the adhesion contact in the stick region is stronger, it is reasonable to assume that the interface energy in the stick phase is smaller than the interface energy 
in the slip phase. We denote this energy difference by $\gamma$. It is clear that without external loading the stick phase is energetically favorable and a finite shear stress is required to get the interface into the slip state.

We first discuss the situation where the external shear stress $\sigma_{x y}$ is given (not the driving velocity). Then we have two homogeneous solutions to our problem: i) uniform stick with pure elastic response and no drift velocity and ii) uniform sliding with drift velocity

$$
v=S \sigma_{x y} / \mu,
$$

where $\mu$ is the shear modulus and $S=\mu / \beta$ is the velocity scale given by the friction law. We note that this homogeneous sliding mode is linearly stable for any velocity with respect to small inhomogeneous perturbations of the stress and strain fields. In this respect the viscous friction law is very different from Coulomb friction which leads to a linear instability and ill-posedness of the problem as has been intensively discussed in the literature [10].

In our previous paper we considered a body uniformly strained with $u_{x y}^{\infty}$ at $x \rightarrow \infty$ and relaxed at $x \rightarrow-\infty$ due to the presence of the slip at $x<0$ (a singular solution of the static theory of elasticity similar to a crack solution, see Fig. 1). The condition for the boundary between semiinfinite slip and stick regions to propagate in the positive $x$-direction is that the energy release because of the stress relaxation is larger than the energy flux to the crack tip (which is proportional to the surface energy):

$$
\Delta=2 \mu\left(u_{x y}^{\infty}\right)^{2} H / \gamma>1
$$

where $H$ is the height of the elastic block. Otherwise, the crack would propagate with negative velocity and the stick phase would be restored. Equilibrium corresponds to

$$
\sigma_{x y}^{\infty}=\sigma_{c} \equiv \sqrt{2 \mu \gamma / H}
$$

The condition $\Delta=1$ or $\sigma_{x y}=\sigma_{c}$ is nothing but the usual Griffith threshold for crack propagation. On the other hand, in the context of the friction problem, this condition may be interpreted as a static friction threshold: a finite shear loading is required to get the system into the sliding mode. The energy released by the creation of a single slip of length $l \gg H / \sqrt{1-2 \nu}$ in the body, uniformly stressed with $u_{x y}$, is $\left(2 \mu\left(u_{x y}\right)^{2} H-\gamma\right) l$. Uniform stick in the long sample is stable if the applied shear stress does not exceed $\sigma_{c}$, otherwise stick is only metastable. We note that for soft materials such as gels the characteristic length $H / \sqrt{1-2 \nu}$ can be quite large. For length scale, shorter than this length, $\sigma_{c}$ increases by factor of 2 compared to Eq. (3). We will return to this point later when accurately solving the elastic problem in Appendix $\mathrm{C}$

On the other hand, the homogeneous sliding mode may be unstable against a resticking pulse (nonlinear "healing instability") if the corresponding value of $\sigma_{x y}<\sigma_{c}$. Since in this case the shear stress is related to the steady-state sliding velocity by Eq. (1), we presume that the homogeneous sliding is stable against the healing instability only above the critical sliding velocity $v_{c}$,

$$
v_{c}=S \sigma_{c} / \mu \text {. }
$$

Thus, for given $\sigma$ we discuss a dynamical first order phase transition between homogeneous stick and slip regimes with $\sigma_{c}$ being the transition point (see Fig. 2). Another

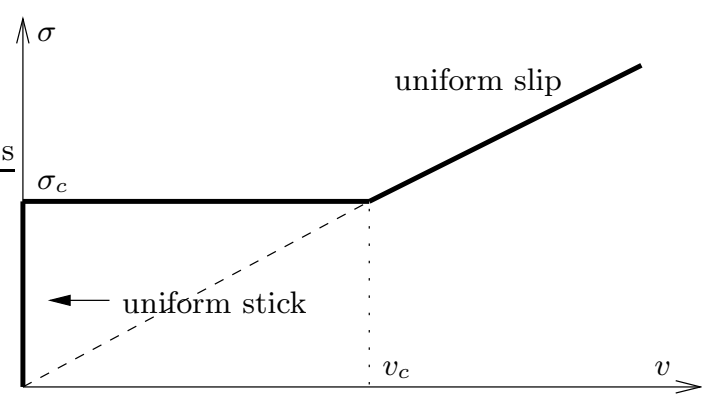

Fig. 2. Two homogeneous regimes, stick and slip; $\sigma_{c}$ is the transition point and also the average stress in stick-slip regime.

example of such a non-equilibrium transition is a dielectric breakdown above some critical value of the applied electric field. The chain reaction of the ionization process takes place forming a conducting state instead of a nonconducting one realized below the critical value of the applied electrical fields. We can also mention a phenomenon of shear banding in complex fluids. Note that these transitions are not real thermodynamical transitions since the slip phase (or conducting state) is not a real thermodynamical phase and dissipation due to friction takes place in this regime. Therefore, our arguments that $\sigma_{c}$ is a transition point are not so strict as in usual thermodynamics. However, we believe that this picture is plausible and also in the spirit of "shear melting" in ultrathin lubricant layers, discussed in 14. In our case, however, the surface properties are strongly coupled to the bulk elasticity and the critical stress $\sigma_{c}$ depends not only on the interface energy difference, $\gamma$, but also on geometry. This is the usual situation in fracture problems and, as we have already mentioned, $\sigma_{c}$ is just the Griffith threshold for crack propagation in this context.

If one starts from the stick phase and increases the stress slightly above the critical value, the transition to the slip regime goes via a nucleation process of slip regions and their growth. Eventually annihilation of the boundaries brings the system into the homogeneous sliding mode. If the sample is not too long, propagation of just one crack may be sufficient to bring the system into the sliding mode. The velocity $c$ of such an isolated crack has been calculated in our previous paper 12:

$$
c=\frac{\pi \ln \Delta}{\varpi \ln (H / a)} S .
$$

Here $\varpi$ is a number related to the Poisson ratio $\nu$ :

$$
\varpi=\frac{3-4 \nu}{2(1-\nu)},
$$

and $a$ is the small length scale cutoff (we will return to this point later). 
If one starts from the sliding mode and reduces the stress below the critical value, the transition to the stick phase is via nucleation and growth of the resticking regions.

We note that, for a given shear stress, this picture does not allow for a steady periodic stick-slip motion. The transition between homogeneous stick and slip states corresponds to the critical value of the stress but, of course, there may be some hysteresis around the transition point, $\sigma_{c}$.

The situation changes drastically when, instead of the shear stress, the driving velocity is prescribed. Actually, most of the experimental setups fix the driving velocity $v$. In this case the uniform stick state is no longer possible. On the other hand, if the driving velocity is below its critical value, given by Eq. (4), uniform sliding may be unstable against resticking pulses (nonlinear "healing instability"). Then we expect the system to exhibit an inhomogeneous sliding mode, namely periodic (quasiperiodic or or even chaotic) stick-slip.

At this point the analogy to the first order phase transitions can be made even deeper: In thermodynamics, if the volume but not the pressure of the system is prescribed for a given temperature, phase coexistence occurs in a range of volumes. If the volume is changed, the pressure remains constant according to the equilibrium phase diagram and only the relative fraction of the two phases changes. If the volume reaches its critical value, one phase disappears and phase equilibrium is not possible for further volume changes. Of course, the driving velocity, which is a dynamical variable, cannot be mapped directly onto the thermodynamical variable, the volume, as well as the hydrostatic pressure is not the same as the shear stress. However, we use this plausible analogy and assume that stick-slip motion (coexistence of two phases) will occur in a range of driving velocities, $v<v_{c}$, with the average shear stress $\sigma \approx \sigma_{c}$, as shown in Fig. 2

Our main goal is to discuss this inhomogeneous mode of sliding. In this paper we analyze this problem only for the steady-state periodic stick-slip motion schematically presented in Fig. 1] The first step is to solve the elastic problem for this geometry and then to calculate the energy flux into the crack tip and the resticking end which allows to formulate equations of motion for these boundaries. This rather technical issue is discussed in detail in the next section. But before some remarks are in order.

The solution of the elastic problems defines a family of the displacement fields and slip velocity fields, each of which is labeled by five parameters: driving velocity $v$, average shear stress $\sigma$, crack velocity $c$, two length scales of the slip and stick regions, $\lambda_{s l}$ and $\lambda_{s t}$ (or alternatively, the wavelength of the pattern $\lambda=\lambda_{s l}+\lambda_{s t}$ and the relative fraction of the slip $\left.\eta=\lambda_{s l} / \lambda\right)$. The driving velocity $v$ is imposed. This defines a problem of a dynamical selection, namely, if a sliding pattern exists, are $c, \lambda_{s l}, \lambda_{s t}$ and $\sigma$ uniquely defined when $v$ is fixed, or not? To find these four parameters we expect to have only two equations of motion for the crack tip and resticking end. Thus, our steady-state periodic problem has a high degeneracy. One needs two additional equations to predict a uniquely selected pattern. Using the analogy to the first order phase transitions we have already removed one degree of freedom, selecting the average shear stress $\sigma$ to be close to its critical value $\sigma_{c}$. However, as in many pattern forming systems the selection of the primary wavelength $\lambda$ is a highly nontrivial issue. In directional solidification and eutectic growth (see, for example, 13]) it is believed that there is only a "soft" selection mechanism which depends on the initial conditions, the history of the process, the level and origin of fluctuations, details of the nucleation and annihilation processes. A detailed discussion of this issue is far beyond the scope of this paper, but we use an additional assumption which leads to a "soft" selection of the wavelength: we assume that the smallest length scale among $\lambda_{s l}$ and $\lambda_{s t}$ is of the order of the sample height $H$.

\section{Solution of the elastic problem and equations of motion for cracks.}

In this Section we solve the problem of linear elasticity with the boundary conditions specified above. We find the expressions for the energy fluxes into the crack edges in terms of the imposed boundary conditions (driving velocity $v$, average shear stress $\tau^{*}=\sigma$, crack tip velocity $c$ ) and geometrical parameters (sample height $H$, pattern wavelength $\lambda=2 \pi / k$ and slip fraction $\left.\lambda_{s l} / \lambda=\eta\right)$. We restrict our attention to the very slow modes assuming that crack velocities are much smaller than the shear wave speed. Stationarity of the problem shows in the fact that the spatial coordinate $x$ enters only in combination with time: $x-c t$. To simplify formulas, we will usually omit the time dependence in the combination.

In Appendix A we consider stationary solutions of the linear elasticity theory in the given geometry and with boundary conditions $\left.u_{y}\right|_{y=0}=\left.u_{y}\right|_{y=H}=\left.\partial_{x} u_{x}\right|_{y=H}=0$. We treat a so called mixed problem of elasticity: there are parts of the bottom surface (slip regions) where only a linear relation between shear strain and stress is given. In the particular case of zero friction, shear stress is zero.

Using the solutions, we can express any relevant quantity (strain or stress components) in terms of the displacement $\left.u_{x}\right|_{y=0}$, or equivalently in terms of the dimensionless sliding velocity $V \equiv \dot{u}_{x} / c=V_{0}-\left.\partial_{x} u_{x}\right|_{y=0}$. The relations are generally nonlocal, leading to an integral equation for the function $V$.

\subsection{Small wavelengths, $\lambda<<H$.}

Let us consider the case where the height is the largest geometrical parameter. Then, as in the case of infinite height, we obtain the following integral equation [1]

$$
\tau(\xi)=\tau^{*}+\frac{2 \mu}{\pi \varpi} \mathrm{P} . \mathrm{V} \cdot \int_{-d}^{d} d \zeta \frac{1+\xi \zeta}{\xi-\zeta} \Phi(\zeta)
$$


where $\tau$ is the shear stress at $y=0$ and

$$
\varpi=\frac{3-4 \nu}{2(1-\nu)} \text {. }
$$

Here we made the variable transformation $\xi=\tan (k x / 2)$, which projects a period $-\pi<k x<\pi$ onto the infinite interval $-\infty<\xi<\infty$, and defined a new function $\Phi(\xi)=$ $V(2 \arctan \xi) /\left(1+\xi^{2}\right)$ which is nonzero in the slip region $|\xi|<d \equiv \tan (\alpha / 2)$. The average dimensionless velocity is given by $V_{0}=v / c$.

First we consider the solutions of the equation in the absence of friction in the slip region $k|x|<\alpha$, which means $\tau(\xi)=0$ for $|\xi|<d\left(\eta=\alpha / \pi=\lambda_{s l} / \lambda\right.$ is the fraction of the slip). The solutions were discussed in Ref. [11] (even with a more complicated form of the viscoelastic bulk response):

$$
\Phi_{0}(\xi)=\frac{\sqrt{1+d^{2}}}{2 \mu} \frac{\varpi \tau^{*} \xi+2 \mu V_{0}}{\left(1+\xi^{2}\right) \sqrt{\left(d^{2}-\xi^{2}\right)}} .
$$

The solution (7) can be rewritten for the strain:

$$
\left.\partial_{x} u_{x}\right|_{y=0}=V_{0}-\frac{\varpi \tau^{*} \tan (k x / 2)+2 \mu V_{0}}{2 \mu \cos (\alpha / 2) \sqrt{\tan ^{2}(\alpha / 2)-\tan ^{2}(k x / 2)}}
$$

in the slip region $|\tan (k x / 2)|<\tan (\alpha / 2)$, and $\left.\partial_{x} u_{x}\right|_{y=0}=$ $V_{0}$ in the stick region. The singularities in the points $|x|=$ $\alpha / k$ are similar to those in a crack solution in $2 d$ elasticity theory. Generally, there is a macroscopic elastic energy flow to a point with the square-root singularity.

The energy flow is determined by the angular dependence of the solution in the vicinity of the point. Thus, we can apply the result for the semi-infinite slip [12] to find the energy flows to the head tip $J_{1}$ and from the rear tip $J_{2}$ of the slip:

$$
J_{1,2}=\frac{\pi c\left(2 \mu V_{0} \pm \varpi \tau^{*} \tan (\alpha / 2)\right)^{2}}{4 \varpi \mu k \tan (\alpha / 2)} .
$$

We see that $J_{2}$ vanishes only if $2 \mu V_{0}-\varpi \tau^{*} \tan (\alpha / 2)=0$, i.e. when the sliding velocity tends to zero near the rear tip. This special solution was discussed in the literature (see, for example, Refs. 11,15]16] and references therein).

The energy flowing to the tips can be reversibly converted into the interface energy and can be dissipated:

$$
J_{1}=\gamma c\left(1+c / c_{0}\right), \quad J_{2}=\gamma c\left(1-c / c_{0}\right),
$$

where $c_{0}$ describes tip dissipation. Let us assume that the tip velocity is so small that the dissipation can neglected. The resulting conditions,

$$
J_{1}=J_{2}=\gamma c
$$

are fulfilled if either $V_{0}=\sqrt{\varpi \gamma k \tan (\alpha / 2) / \mu}$ and $\tau^{*}=0$, or $\tau^{*}=2 \sqrt{\gamma \mu k /(\varpi \tan (\alpha / 2))}$ and $V_{0}=0$. The latter case corresponds to the periodic array of slip regions moving with velocity $c$; in the co-moving frame the strain distribution is the same as in the statics. This solution is unstable. For example, for the fixed wave number $k$ and stress smaller than its equilibrium value, $\alpha$ will decrease until the stick conditions hold everywhere. The former solution has the feature that the sliding preserves its direction $(V>0)$ in the whole slip region.

The case with non-zero friction can be treated as above. Now, in the left-hand side of Eq. (6), in the slip region $|\xi|<d$, we have $\tau(\xi)=\mu c\left(1+\xi^{2}\right) \Phi(\xi) / S$. The solution can be guessed from that for the semi-infinite slip 12]:

$$
\Phi(\xi)=\frac{b_{1} \xi+b_{2}}{\left(1+\xi^{2}\right)(\xi+d)^{(1+\epsilon) / 2}(d-\xi)^{(1-\epsilon) / 2}},
$$

where $b_{1}, b_{2}$ and $\epsilon$ are given by the following expressions:

$$
\begin{aligned}
& b_{1}=\frac{\cos \frac{\pi \epsilon}{2}}{2 \mu \cos (\alpha / 2)}\left(-2 \mu V_{0} \sin \frac{\epsilon \alpha}{2}+\varpi \tau^{*} \cos \frac{\epsilon \alpha}{2}\right), \\
& b_{2}=\frac{\cos \frac{\pi \epsilon}{2}}{2 \mu \cos (\alpha / 2)}\left(2 \mu V_{0} \cos \frac{\epsilon \alpha}{2}+\varpi \tau^{*} \sin \frac{\epsilon \alpha}{2}\right), \\
& \tan \frac{\pi \epsilon}{2}=\frac{\varpi c}{2 S} .
\end{aligned}
$$

Elastic energy flow in the presence of friction $\sigma_{x y} \propto \dot{u}_{x}$ depends 12 on the distance from the tip $r$ :

$$
J_{1}=\frac{\mu c^{2}}{S \epsilon} K^{2} r^{\epsilon}
$$

where $K$ is a coefficient in front of the main contribution in the strain $\left|\partial_{x} u_{x}\right|=K|r|^{(-1+\epsilon) / 2}$ near the tip in the slip region at $y=0$. In the expression for the rear tip $\epsilon$ is replaced by $-\epsilon$. It can be easily seen that on each scale the change of the elastic energy flow equals the energy loss due to friction. Thus, we must allow that on the microscopic scale $a$ the friction law is changed so that the elastic energy flow on smaller scales remains constant, the same as for $r=a$. For example, this should be the case, if the frictional shear stress saturates at large velocities. We assume that this microscopic cutoff $a$ is a material property which can be considered to be the same for the head and rear tips and essentially is independent of $\epsilon$. Then we can express the energy flows in terms of $V_{0}, \tau^{*}$ and $\epsilon$ :

$J_{1}=\frac{c\left(2 \mu V_{0} \cos \frac{(1+\epsilon) \alpha}{2}+\varpi \tau^{*} \sin \frac{(1+\epsilon) \alpha}{2}\right)^{2}(k a)^{\epsilon}}{(2 \sin \alpha)^{1+\epsilon} \varpi \mu k} \frac{\sin (\pi \epsilon)}{\epsilon}$,

$J_{2}=\frac{c\left(2 \mu V_{0} \cos \frac{(1-\epsilon) \alpha}{2}-\varpi \tau^{*} \sin \frac{(1-\epsilon) \alpha}{2}\right)^{2}(k a)^{-\epsilon}}{(2 \sin \alpha)^{1-\epsilon} \varpi \mu k} \frac{\sin (\pi \epsilon)}{\epsilon}$.

We argue that if the height is larger than either of the lengths $\lambda_{s l}$ or $\lambda_{s l}$ and smaller than the other one, the solutions for large height are valid essentially. Minor changes can be determined with the help of the solutions for isolated stick and slip regions given in Appendix B

\subsection{Large wavelengths, $\lambda>>H$.}

In the limit of long slip and stick regions, one obtains "zero-mode" solutions with the relaxed stresses modified 
by the presence of friction. The solutions are valid far from the slip edges. In the stick region $|x|<\lambda_{s t} / 2$,

$$
\begin{aligned}
& u_{x}=V_{0} \frac{(H-y) x}{H}+\frac{\sigma_{0} y}{\mu}, \quad u_{y}=V_{0} \frac{y(y-H)}{4(1-\nu) H}, \\
& \sigma_{x y}=\sigma_{0}-\mu \frac{x}{H} V_{0}
\end{aligned}
$$

and the normal stresses $\sim \mu V_{0} /(1-2 \nu)$ depend only on $y$. Here $\sigma_{0}$ is related to the displacement $u_{0}$ of the sample top with respect to the middle of the stick: $\sigma_{0}=\mu u_{0} / H$.

In the slip region shear stress is relaxed to $\mu v / S$ and the sliding velocity is equal to the driving velocity $v$ with exponentially small corrections far from the boundary points.

Since, in the limit of small height, the normal stresses are relaxed in the slip region, we can find the energy fluxes similarly to the case of semi-infinite slip [12].

Using the asymptotic solutions described above, we get energy flows to (from) the regions of length $\sim H$ surrounding boundary points:

$$
J_{1,2}^{(0)}=\frac{\mu c H}{2}\left(\frac{\sigma_{0}}{\mu} \pm \frac{\lambda_{s t}}{2 H} V_{0}\right)^{2}
$$

where the subscripts 1 (upper sign) and 2 (lower sign) correspond to the regions near the rear and head tips of the stick region, respectively.

Without friction, inverse-square-root singularities appear in the strains and stresses within a distance $\sim H$ around the boundary points. The friction changes the exponents to $-1 / 2 \pm \epsilon$ and creates additional dissipative energy flows from the sample. These dissipative flows are also restricted within the distance $\sim H$ in the slip regions.

Thus, the expressions for the energy flows, instead of Eq. (16), take the following form in the limit of small height:

$$
\begin{aligned}
& J_{1}=\frac{\mu c H}{2}\left(\frac{\sigma_{0}}{\mu}+\frac{\lambda_{s t}}{2 H} V_{0}\right)^{2}\left(\frac{H}{a}\right)^{-\epsilon}, \\
& J_{2}=\frac{\mu c H}{2}\left(\frac{\sigma_{0}}{\mu}-\frac{\lambda_{s t}}{2 H} V_{0}\right)^{2}\left(\frac{H}{a}\right)^{\epsilon} .
\end{aligned}
$$

These equations can be rewritten in terms of the average shear stress $\tau^{*}$ using the relation

$$
\sigma_{0}=\frac{\lambda}{\lambda_{s t}}\left(\tau^{*}-\mu \frac{v}{S}\right)
$$

Strictly speaking, if $1-2 \nu \ll 1$, the relations derived here are not valid on intermediate scales $H \ll \lambda_{s t}, \lambda_{s t} \ll$

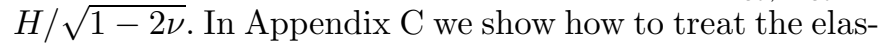
tic problem on all scales larger than $H$ without friction. The results on intermediate scales do not substantially differ from those obtained above (only by numerical factors if written in terms of $\tau^{*}$ ).

\subsection{Stability and degeneracy}

Solving the elastic problem we have found energy flows to the boundary points of slip pulses $J_{1,2}$. Equations of motion for the tip and the resticking end of the crack are given by the energy conservation, Eqs. (10). Therefore, we cannot determine four quantities $\left(c, \tau^{*}, k=2 \pi / \lambda\right.$, $\left.\eta=\alpha / \pi=\left(\lambda-\lambda_{s t}\right) / \lambda\right)$ using only two equations. Two of the parameters cannot be chosen directly, and we are faced with the problem of dynamical selection. We will address this issue in the next section using stability arguments.

There is another kind of degeneracy related to the fact that the sign of the term in the parentheses in Eqs. (16) and (20) for $J_{2}$ can be either negative or positive, depending on relative strength of the average shear stress and velocity. For simplicity, we consider this question in the case of large wavelengths. For small wavelengths, the conclusion will be similar. In the following we neglect the tip dissipation $\left(c / c_{0}<<1\right)$ assuming that the main dissipation comes from the friction in the slip region. Then Eqs. (10) reduce to $J_{1}=J_{2}=\gamma c$. The velocities of the rear tip $\left(c_{1}\right)$ and the head tip $\left(c_{2}\right)$ of the stick region are (see Eq. (5) and (20) )

$$
c_{1}=\frac{\pi S \ln \Delta_{1}}{\varpi \ln (H / a)}, \quad c_{2}=-\frac{\pi S \ln \Delta_{2}}{\varpi \ln (H / a)},
$$

where

$$
\Delta_{1,2}=\frac{H}{2 \mu \gamma}\left(\frac{\mu V_{0} \lambda_{s t}}{2 H} \pm \sigma_{0}\right)^{2}
$$

For the steady-state motion $c_{1}=c_{2}=c$ and $V_{0}=v / c$. There are two branches of the solution. On the first branch, we have:

$$
\left(\frac{\mu V_{0} \lambda_{s t}}{2 H}\right)^{2}-\sigma_{0}^{2}=\sigma_{c}^{2}
$$

thus $\mu V_{0} \lambda_{s t} /(2 H) \geq \sigma_{c}$, and $\sigma_{0}$ can be arbitrarily small.

On the second branch,

$$
\sigma_{0}^{2}-\left(\frac{\mu V_{0} \lambda_{s t}}{2 H}\right)^{2}=\sigma_{c}^{2}
$$

This branch contains the special static solution $c=v=0$, $V_{0}=0$ for $\sigma_{0}=\sigma_{c}$. This solution is unstable with respect to a change of $\lambda_{s t}$. The instability can be seen from the fact that the equilibrium corresponds to the energy maximum. On the contrary, for the special static solution with $\sigma_{0}=$ $0, c=0$ on the first branch, the total energy of the stick is dominated for small $\lambda_{s t}$ by the surface energy $-\gamma \lambda_{s t}$, and for large $\lambda_{s t}$ by the elastic energy

$$
H \int_{-\lambda_{s t} / 2}^{\lambda_{s t} / 2} d x \frac{1}{2 \mu}\left(\frac{\mu V_{0} x}{H}\right)^{2}=\frac{\mu V_{0}^{2} \lambda_{s t}^{3}}{24 H},
$$

and hence there must be a minimum of the total energy corresponding to the stable equilibrium. 
A more general stability criterion can be derived as follows. Consider the effect of a small change of the stick length $\delta \lambda_{s t}$. We suppose that resticking occurs with the same strain $V_{0}$. The change of the stick length leads to the changes of the tip velocities:

$$
\begin{gathered}
\delta c_{1}=\frac{2 \pi S}{\varpi \ln (H / a)} \frac{\mu V_{0}}{\mu V_{0} \lambda_{s t}+2 H \sigma_{0}} \delta \lambda_{s t}, \\
\delta c_{2}=-\frac{2 \pi S}{\varpi \ln (H / a)} \frac{\mu V_{0}}{\mu V_{0} \lambda_{s t}-2 H \sigma_{0}} \delta \lambda_{s t},
\end{gathered}
$$

and hence,

$$
\frac{\delta c_{2}-\delta c_{1}}{\delta \lambda_{s t}}=-\frac{2 \pi S}{\varpi \ln (H / a)} \frac{2\left(\mu V_{0}\right)^{2} \lambda_{s t}}{\left(\mu V_{0} \lambda_{s t}\right)^{2}-\left(2 H \sigma_{0}\right)^{2}} .
$$

The necessary stability condition $\left(\delta c_{2}-\delta c_{1}\right) / \delta \lambda_{s t}<0$ is satisfied only on the first branch.

\section{Dynamical selection of the stick-slip pattern}

We note that no deterministic approach can fix the two free parameters which appear in the result. In this section, we discuss the selection problem for the parameters of the stick-slip pattern on the example of a long sample, starting from the limit of small driving velocities.

We focus our attention on the first stable branch. For small heights, different stick regions do not interact, and each of them is characterized by its length $\lambda_{s t}$, propagation velocity $c$ and shear stress parameter $\sigma_{0}$ (see Eqs. (2224)):

$$
\begin{aligned}
& \left(\frac{\mu V_{0} \lambda_{s t}}{2 H}\right)^{2}-\sigma_{0}^{2}=\sigma_{c}^{2}, \quad V_{0}=\frac{v}{c}, \\
& c=\frac{2 \pi S}{\ln (H / a)} \ln \left(\frac{\sqrt{\sigma_{c}^{2}+\sigma_{0}^{2}}+\sigma_{0}}{\sigma_{c}}\right) .
\end{aligned}
$$

In the exactly stationary regime $c$ is the same for different stick regions. The solutions $\left(c, \lambda_{s t}\right)$ can be parametrized by $\sigma_{0}$. The wavelength $\lambda$ is a free parameter.

It is plausible to assume that $\sigma_{0}$ cannot be much larger than $\sigma_{c}$. The inequality $\sigma_{0} \gg \sigma_{c}$ would lead to large values of the shear stress $\sigma \gg \sigma_{c}$ in regions of the length exceeding $H$. Such a region is unstable with respect to slip. Larger values of $\sigma_{0}$ are not impossible, they are merely less probable. Thus, we can find an upper estimate for the crack tip velocity $c_{\max }$ :

$$
c_{\max } \sim \frac{2 \pi S}{\ln (H / a)},
$$

and a relation for the stick length:

$$
\lambda_{s t} \sim \frac{2 H c \sigma_{c}}{\mu v}<\lambda_{s t, \max } \sim \frac{4 \pi H S \sigma_{c}}{\mu v \ln (H / a)} .
$$

We see that the upper limit for $\lambda_{s t}$ vanishes with increasing $v$. In the case of small heights, only $\lambda_{s t}>H$ is possible and hence $v<v^{*}$, where

$$
v^{*} \sim \frac{4 \pi S \sigma_{c}}{\mu \ln (H / a)}=\frac{4 \pi v_{c}}{\ln (H / a)} .
$$

Concerning the value of $\lambda_{s t}$, two cases can be considered. The first possibility is to have $c \sim c_{\max }$ and $\lambda_{s t} \sim \lambda_{s t, \max }$.

The second possibility following from Eq. (30) is $c \sim$ $c_{\max }\left(\sigma_{0} / \sigma_{c}\right) \ll c_{\max }$ corresponding to $\sigma_{0} \ll \sigma_{c}$, which would lead to

$$
\lambda_{s t} \sim \frac{4 \pi H S \sigma_{0}}{\mu v \ln (H / a)} \ll \lambda_{s t, \max } .
$$

If long stick regions are present in the sample, they will eventually catch up with shorter and slower stick regions, and then probably merge with them. Consequently, it is plausible that the lengths of the stick regions are mostly of order of $\lambda_{s t, \max }$, specified in Eq. (32), and $\tau^{*} \sim \sigma_{c}$. As $v$ decreases, this length diverges.

On the other hand, the length $\lambda_{s l}$ should be of the order of $H$ for small $v$ because large slip regions, $\lambda_{s l}>>$ $H$ with small frictional stress would be unstable against resticking. The size of the critical nucleus of the slip inside the stick phase with $\sigma_{0} \sim \sigma_{c}$ is of the order of $H$ which also prevents $\lambda_{s l}$ to be much smaller than $H$.

On the opposite side of the stick-slip regime as $v$ approaches $v_{c}$, the same arguments lead to $\lambda_{s t} \sim H$. Thus, we can employ the condition

$$
\min \left\{\lambda_{s t}, \lambda_{s l}\right\} \sim H
$$

This is fully compatible with the condition

$$
\tau^{*} \sim \sigma_{c},
$$

which we have found in the small velocity limit and also using the analogy to the two-phase region in the first order phase transitions.

The results shown in Figures 3 - 5 represent numerical solutions of the equations of motion, $J_{1}=\gamma c$ and $J_{2}=\gamma c$, together with two additional constraints (35136). We model the first condition by the requirement that $k H / \sin \alpha$ is a fixed number. The dependences of the slip fraction and the dimensionless crack tip velocities on the driving velocity are evaluated both for the limits of large and small heights. In Fig. 5 the values of $\lambda_{s t}$ and $\lambda$ are given in units of $H$. The crack velocities $c$ remain of the order of $S$ for all values of $v / v_{c}$, the fraction of slip $\eta$ gradually changes from 0 to 1 and the wavelength $\lambda$ diverges at the both ends of the stick-slip region being still of the order of $H$ for the intermediate values of $v / v_{c}$.

The sliding velocity in the periodic stick-slip regime calculated from Eqs. (11.13) is presented in Fig. [6 for a certain value of the driving velocity. Note that the singularities at the head and the rear of the slip pulses are quite different.

\section{Discussion}

A low velocity dynamics consisting of periodic slip pulses which heal at a critical value of the local slip velocity is usually attributed to the existence of a V-weakening frictional regime [5]. In this unstable regime the sliding velocity decreases with the increase of the stress. Our model 


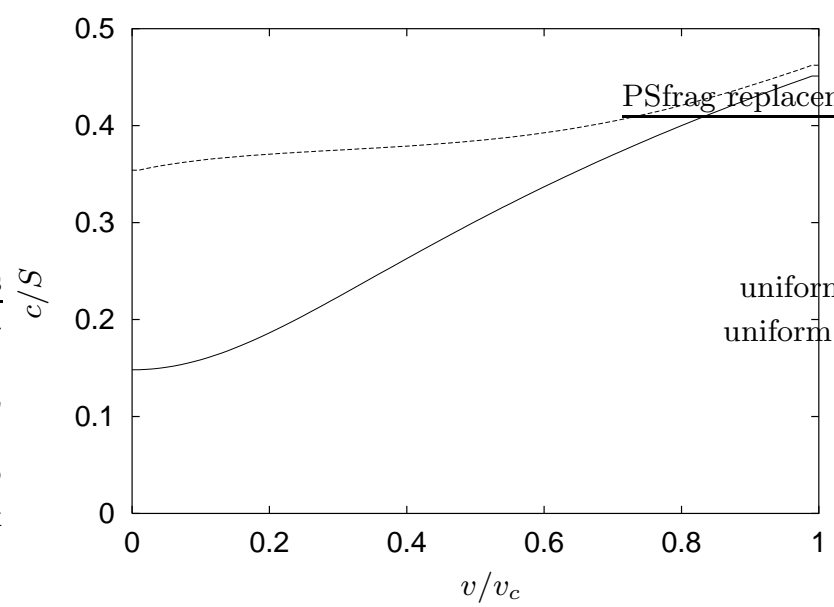

Fig. 3. Ratio of the crack propagation velocity to the velocity scale $S$ set by viscous friction as a function of $v / v_{c}$ for two values of $k H /(\pi \sin \alpha): 2$ (solid line) and 0.4 (dashed line); For all plots $\pi a /(2 H)$ was $2 \cdot 10^{-6}$.

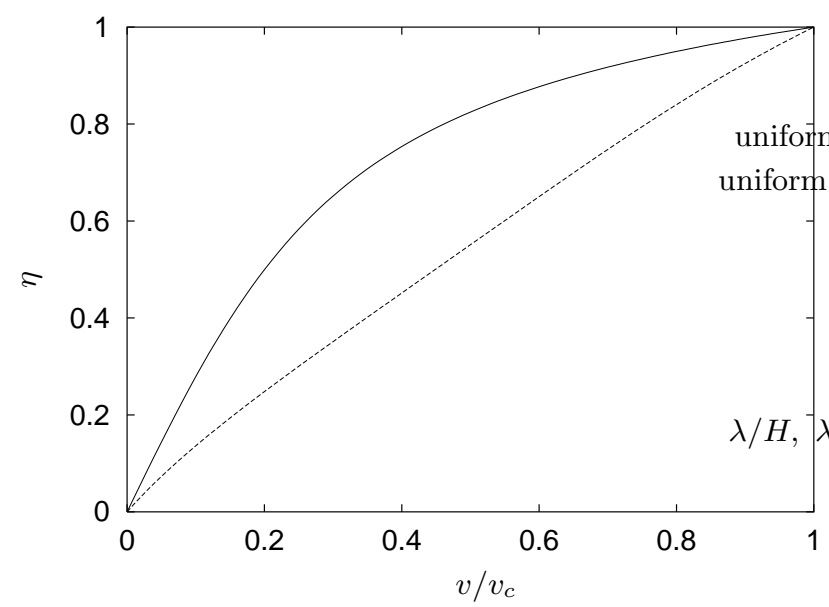

Fig. 4. Fraction of the $\operatorname{slip} \eta=\alpha / \pi$ as a function of $v / v_{c}$ for two values of $k H /(\pi \sin \alpha): 2$ (solid line) and 0.4 (dashed line); $\eta$ does not strongly depend on $k H /(\pi \sin \alpha)$. For all plots $\pi a /(2 H)$ was $2 \cdot 10^{-6}$.

does not contain intrinsic instabilities of the friction law as a $\mathrm{V}$-weakening frictional regime. The homogeneous slip is still metastable below the critical velocity in our picture. Critical shear stress and critical velocity depend not only on material properties but also on the geometry and they are related to the point of discontinuous transition (to the Griffith threshold for crack propagation). These two scenarios do not contradict each other and can be combined in one model (see, for example, 14 for a description of "shear induced melting"). This is the usual situation in first order phase transitions: both, the transition point and instability points exist, forming the metastable regions around the transition point. The characteristic velocity scale for the discontinuous transition and velocity scale for a $\mathrm{V}$-weakening unstable frictional regime can be well separated leading to stick-slip even above the instability point but, of course, below the transition velocity. In

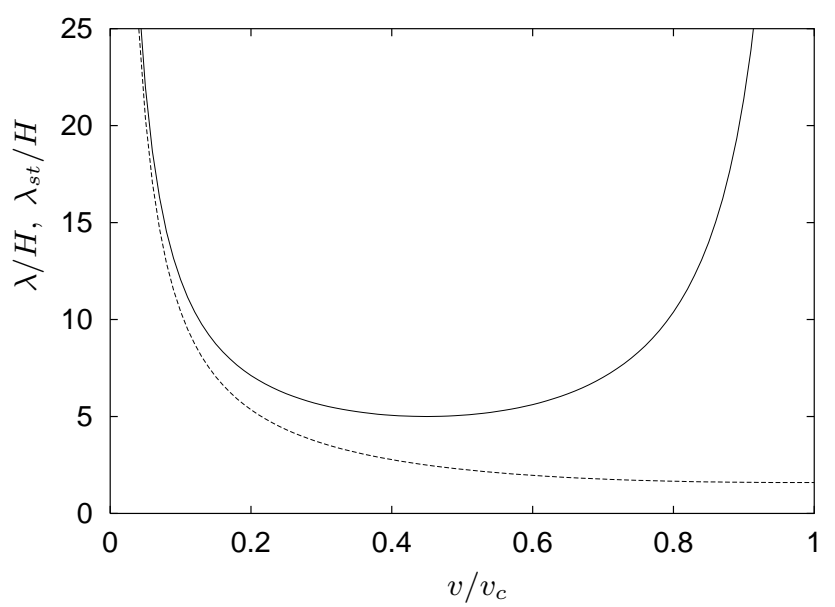

Fig. 5. $\lambda / H$ (solid line) and $\lambda_{s t} / H$ (dashed line) as functions of $v / v_{c}$ for $k H /(\pi \sin \alpha)=0.4$. For all plots $\pi a /(2 H)$ was $2 \cdot 10^{-6}$.

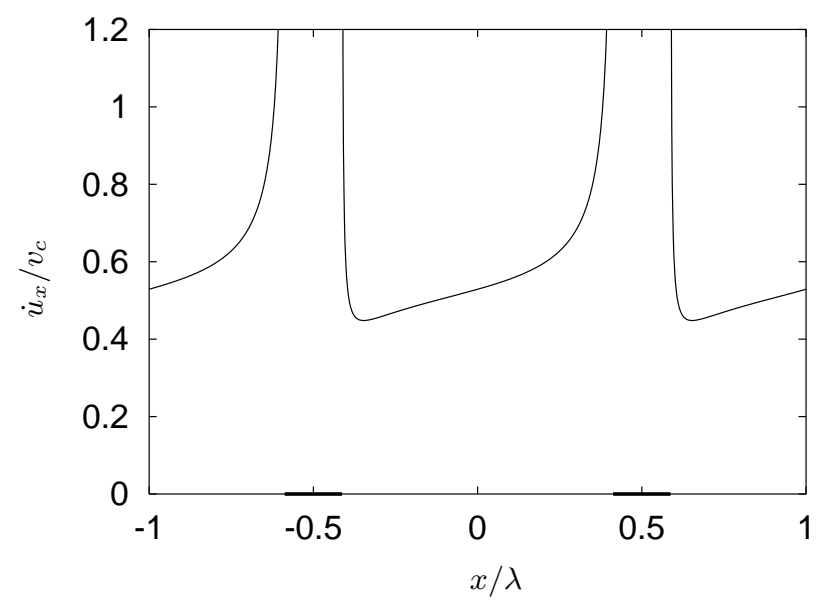

Fig. 6. Dimensionless sliding velocity $\left.\dot{u}_{x}\right|_{y=0}$ in the periodic stick-slip regime as a function of the coordinate for $k H /(\pi \sin \alpha)=2$. Two periods are shown. The sliding velocity is zero in the stick regions around $|x|=\lambda / 2$. Pattern parameters are calculated for the driving velocity $v=0.5 v_{c}$.

our model we discuss the limiting situation where the Vstrengthening regime holds even for very small velocities (at least smaller than our transition velocity.

Now let us discuss the relation of the obtained results to the very interesting experimental observations of Baumberger, Caroli and Ronsin 4,5. They performed experiments of a gel sliding on a glass plate. The driving velocity was given and the shear force, and thus the average shear stress, was deduced from the spring elongation. Above some critical driving velocity $v_{c} \approx 100 \mu \mathrm{m} / \mathrm{s}$, steady sliding was observed. At velocities smaller than the critical one, periodic stick-slip sets in (see figures in [4, 5]). Upon decreasing the driving velocity $v$, no hysteresis of the transition was detected. In the stick-slip regime they observed the propagation of self-healing pulses with no opening, nucleated periodically at the trailing edge of the sample. The propagation velocity of these cracks was about 60 times larger than the critical sliding velocity, yet 
still much smaller than the shear wave speed. The cracklike singularities in the slip velocity field were detected behind the tip of the pulse but not at the resticking end.

The authors very carefully studied the dependence of the critical velocity, crack velocities and other properties of slip pulses on the properties of the gel, but the dependence on geometry was not discussed. Since both the critical velocity and the critical stress, predicted by our model, depend on the height of the sample and not only on material parameters, the crucial test for the relevance of our model to this experiment is not possible yet. Moreover, we assumed a very large sample length compared to its height and discuss a spatially periodic stick-slip pattern, while in the experiment at most one slip pulse was simultaneously observed in the sample nucleated at the trailing edge. The length of the slip pulse increased with the driving velocity $v$ and sometimes the sample was too short to observe more than one stick-slip boundary. Furthermore, the fact, that no hysteresis of the transition was detected in the experiment, points towards the possible interference of the intrinsic instabilities for low sliding velocities, which have not been discussed in our model so far. However, as we have already mentioned, the characteristic velocity scale for the discontinuous transition and the velocity scale for the $\mathrm{V}$-weakening unstable frictional regime can be made well separated, if smaller heights of the sample would be available for experimental investigation.

The observed nonlinear behavior of the stress with the velocity for relatively high sliding rates (the so-called shear-thinning rheology) can, in principle, be incorporated into a more sophisticated version of the theory presented here. This has already been discussed shortly in the context of the microscopic cutoff $a$, the small region around the crack edge where the transition from stick to slip takes place.

Another way to approach the observed dependence of the shear stress on the sliding velocity is to make a simple modification of the friction law in the slip region which amounts to the addition of the constant: $\sigma_{x y}=$ const + $\beta \dot{u}_{x}$. This modification does not lead to any changes in our results except for a trivial shift of all values of the shear stresses.

The predictions of our theory may differ from experimental results due to different geometries, interference of the intrinsic instabilities, some specific features caused by polymeric nature of the gel etc. The effects on the propagation of single slip pulses are planned to be considered elsewhere 17.

Our aim was to construct a generic, conceptually simple model which allows for stick-slip motion in sliding friction without call to some very specific properties of materials. In our model all complicated properties of the gel are hidden in only two material parameters which are related to surface properties: the energy scale $\gamma$ and the velocity scale $S$ due to the viscous frictional law. Of course, these parameters may depend on the applied normal stress but we assume that they exist also for zero normal stress. The velocity scale $S$ can be easily estimated from the shear stress response to a jump of the driving velocity in the sliding regime (see Fig. 15 in [5]). This response is pure linear in our model and leads to

$$
\sigma(t)=\sigma_{f i n}-\left(\sigma_{f i n}-\sigma_{i n}\right) \exp (-S t / H)
$$

where $\sigma_{i n}$ and $\sigma_{\text {fin }}$ are the initial and final stresses before and after the jump. The response is not changed if one takes a modified friction law by adding a constant as described above. This exponential behavior is in a good agreement with the experimental curve for large changes of stresses and velocities (see Fig. 17) and gives an estimate for $S / H \approx 1 \mathrm{~s}^{-1}$, which leads to $S \approx 1 \mathrm{~cm} / \mathrm{s}$ for used samples with $H=1 \mathrm{~cm}$.

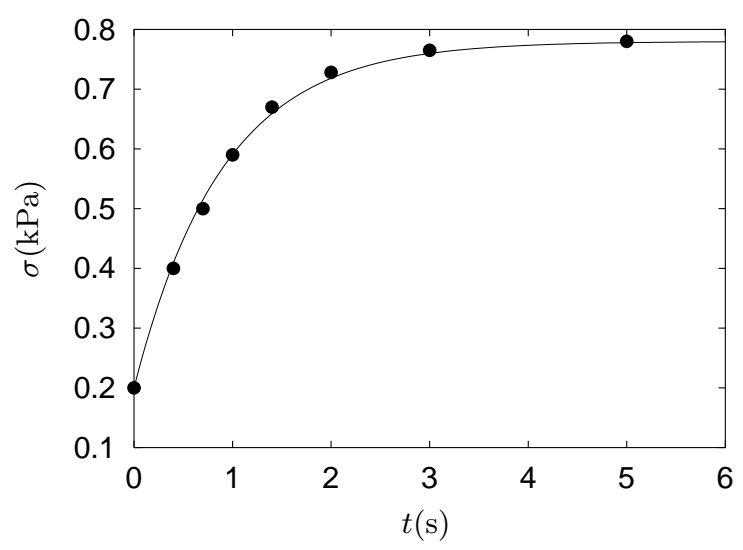

Fig. 7. Several experimental points taken from Fig. 15 of Ref. [5]: relaxation of shear stress after a jump of the driving velocity. The line represents a fit according to Eq. (37).

One would expect that for ordinary elastic materials the velocity $S$ should be of the order of the speed of sound. However, for gels the shear modulus $\mu$ is much smaller than for ordinary materials. The shear sound velocity $c_{t}=$ $(\mu / \rho)^{1 / 2}$ is only $2 \mathrm{~m} / \mathrm{s}$. The velocity $S=\mu / \beta$ is linear in $\mu$ and should be even smaller. This is a possible explanation for a relatively small value of $S$ compared to $c_{t}$.

The existence of a critical sliding velocity, where the transition to a stationary sliding occurs, appears naturally in our description and is given by Eqs. (34). The length of the slip pulses increase as $v$ approaches $v_{c}$, and the fraction of the slip phase tends to unity in qualitative agreement with the experiments. The characteristic value of the shear strain in the sliding mode near the critical velocity experimentally was about $u_{x y}=0.04$. Thus, we can estimate from Eq. (3) the characteristic difference between the interface energies in the slip and stick states to be $\gamma=0.1 \mathrm{~J} / \mathrm{m}^{2}$.

In the stick-slip regime, which exists below $v_{c}$, the nucleation of a slip pulse takes place at the trailing edge of the sample and requires overshooting above the Griffith threshold according to the experiment. This overshooting is not small and we can use Eq. (5) to estimate the crack velocity. Because of the week logarithmic parameter dependence, we conclude that the crack propagation velocity $c$ is of the order of $S$ and essentially independent of 
the driving velocity, in agreement with experimental observations. After the slip pulse traversed the sample the stress drops below the Griffith threshold and resticking takes place via propagation of a healing front. Theoretical predictions for the crack velocities $c$ in the steady-state spatially periodic stick-slip regime are given in Fig. 3 we get $c \sim S \approx 1 \mathrm{~cm} / \mathrm{s}$, which is also in qualitative agreement with the experiments.

The crack-like singularities in the slip velocity field are predicted by our theory and were detected behind the tip of the pulse. At the resticking end the sliding velocity in the experiment suddenly drops to zero from a finite value which is close to the critical velocity. We should note that this discontinuity with finite amplitude is not compatible with solutions of the elastic problem which allow either crack-like singularities or, as a particular solution, a smooth, continuous termination of the slipping process at the healing point. This type of solutions, which correspond to a vanishing stress intensity factor due to some additional constraint on the parameters, was discussed in the literature (e.g. Refs. 15 16]) and also in the previous sections. Perhaps, one could argue that a fast transient, too fast to be experimentally resolved, takes place (see Fig. 6).

The geometry of the experiment is such that the total macroscopic friction of the sliding sample and the nucleation of the pulses depend on the processes taking place at the edges of the sample. The stresses here are highly inhomogeneous and the kinetic phenomena should be considered with a great care. It would be very interesting to perform a experiment in such conditions where the length of the sample and its height are well separated and to study the possible height dependence of the critical velocity and the shear stress. We believe that further theoretical and experimental investigations will shed light on this phenomenon where two intriguing problems, crack propagation and friction, combine together.

We would like to acknowledge fruitful discussions with E. I. Kats, J. S. Langer, and V. Steinberg. We are grateful to H. Schober for reading the manuscript and useful comments. S.M. and V.M. thank the Forschungszentrum Jülich for its hospitality. S.M. thanks RFBR for financial support under grant No. 03-02-16173, and DFG for support in the framework of SFB 608.

\section{A Relation between strain and stress at $y=0$}

We consider only periodic stationary solutions, possible generalizations are specially mentioned.

A starting point is the momentum conservation equation of the linear elasticity theory:

$$
\partial_{t}^{2} u_{\alpha}=\left(c_{l}^{2}-c_{t}^{2}\right) \nabla_{\alpha} \nabla_{\beta} u_{\beta}+c_{t}^{2} \nabla^{2} u_{\alpha},
$$

where $c_{l}$ and $c_{t}$ are the longitudinal and transverse sound velocities respectively, $c_{l} / c_{t}=\sqrt{2(1-\nu) /(1-2 \nu)}$.
We consider only the plane strain case with $u_{z}=0$. The solutions can be found in form of Fourier series [11]:

$$
\begin{aligned}
u_{x}= & v t+u_{x}^{*}(y)+\operatorname{Re} \sum_{m=1}^{\infty}\left(a_{m} e^{-m s_{-} y}+b_{m} e^{-m s_{+} y}\right. \\
& \left.+c_{m} e^{m s_{-} y}+d_{m} e^{m s_{+} y}\right) e^{i m k(x-c t)} \\
u_{y}= & \operatorname{Re} \sum_{m=1}^{\infty}\left(a_{m} \frac{i k}{s_{-}} e^{-m s_{-} y}+\frac{i s_{+}}{k} b_{m} e^{-m s_{+} y}\right. \\
& \left.-\frac{i k}{s_{-}} c_{m} e^{m s_{-} y}-\frac{i s_{+}}{k} d_{m} e^{m s_{+} y}\right) e^{i m k(x-c t)}, \\
\sigma_{x y}= & \tau^{*}+\mu \operatorname{Re} \sum_{m=1}^{+\infty} m k\left(-a_{m}\left(\frac{k}{s_{-}}+\frac{s_{-}}{k}\right) e^{-m s_{-} y}\right. \\
& -2 \frac{s_{+}}{k} b_{m} e^{-m s_{+} y}+\left(\frac{k}{s_{-}}+\frac{s_{-}}{k}\right) c_{m} e^{m s_{-} y} \\
& \left.+2 \frac{s_{+}}{k} d_{m} e^{m s_{+} y}\right) e^{i m k(x-c t)},
\end{aligned}
$$

where

$$
s_{+}=k \sqrt{1-\frac{c^{2}}{c_{l}^{2}}}, \quad s_{-}=k \sqrt{1-\frac{c^{2}}{c_{t}^{2}}},
$$

and $k$ is the pattern wave number: $k=2 \pi / \lambda$. In a periodic pattern, an average value of a quantity corresponds to its zero Fourier harmonic. We assume that on average there is no normal stress, i.e. $u_{x}^{*}(y)$ is a linear function, and there is no zero-harmonic term in $u_{y}$.

We use boundary conditions $\left.u_{y}\right|_{y=0}=\left.u_{y}\right|_{y=H}=0$ and $\left.\partial_{x} u_{x}\right|_{y=H}=0$, which give three linear uniform equations for $a_{m}, b_{m}, c_{m}, d_{m}$. This allows to express the stress $\left.\tau \equiv \sigma_{x y}\right|_{y=0}$ in terms of the Fourier harmonics of the dimensionless sliding velocity $V \equiv \dot{u}_{x} / c=V_{0}-\partial_{x} u_{x}$ :

$$
\tau(\eta)=\tau^{*}+\mu \operatorname{Re} \sum_{m=1}^{\infty}(-2 i) \chi(m) B_{m} e^{i m k(x-c t)},
$$

where $B_{m}$ is

$$
B_{m}=\frac{1}{\pi} \int_{-\pi / k}^{\pi / k} d x k V(x) e^{-i m k x},
$$

and $\chi(m)$ is calculated under the assumption $c^{2} \ll c_{t}^{2}, c_{l}^{2}$ :

$$
\chi=2(1-\nu) \frac{(3-4 \nu) \sinh (m k H) \cosh (m k H)-m k H}{(3-4 \nu)^{2} \sinh ^{2}(m k H)-(m k H)^{2}} .
$$

This is the result of the static elasticity theory. The first $c$-dependent correction would be proportional to $c^{2} / c_{t}^{2}$.

We note that the limit of $\nu \rightarrow 1 / 2$ and $m k H \rightarrow 0$ is not well defined: the limiting value of $\chi$ depends on the ratio $(m k H)^{2} /(1-2 \nu)$. If $(m k H)^{2} \ll(1-2 \nu)$ one gets $\chi(m)=1 /(2 m k H)$. The relation between zero harmonics of the shear strain and the stress $\tau=2 \mu u_{x y}$ is recovered on scales larger than $H / \sqrt{1-2 \nu}$. If $\nu$ is close to $1 / 2$, there 
is an intermediate region $\sqrt{1-2 \nu} \ll m k H \ll 1$ where we have $\chi(m) \approx 2 /(m k H)$. Finally, if $m k H \gg 1$,

$$
\chi=\frac{2(1-\nu)}{3-4 \nu} \equiv \frac{1}{\varpi} .
$$

The latter is realized for the periodic pattern with the wavelength smaller than the height, and all the terms in the Fourier series satisfy the condition $m k H \gg 1$.

\section{B Short isolated stick and slip regions}

In this Appendix we specify the results for short isolated slip and stick regions. This limit is characterized by the following relation between the strain (expressed in terms of dimensionless velocity $V$ in the stationary regime) and the stress $\left.\tau(x) \equiv \sigma_{x y}\right|_{y=0}$ :

$$
\tau(x)=\frac{2 \mu}{\pi \varpi} \mathrm{P} \cdot \mathrm{V} \cdot \int \frac{d z}{x-z} V(z)+\tau^{*},
$$

which is derived similarly to the periodic case.

\section{B.1 Single slip of length $\lambda_{s l} \ll H$}

First consider the case of a short slip, propagating with speed $c$, in the presence of friction proportional to sliding velocity; the top surface is uniformly displaced and fixed (no driving velocity).

The solution with zero friction which satisfies the condition $V=0$ in the stick region $|x|>\lambda_{s l} / 2$ has the following form:

$$
V(x)=\left\{\begin{array}{rr}
\frac{b_{1} x+b_{2}}{\sqrt{\left(\lambda_{s l} / 2\right)^{2}-x^{2}},} & |x|<\lambda_{s l} / 2 \\
0, & |x|>\lambda_{s l} / 2
\end{array},\right.
$$

where

$$
\begin{aligned}
& b_{1}=\frac{\varpi \tau^{*}}{2 \mu}, \quad b_{2}=\frac{1}{\pi}\left(u_{2}-u_{1}\right), \\
& u_{1}=\left.u_{x}\right|_{x=\lambda_{s l} / 2}, \quad u_{2}=\left.u_{x}\right|_{x=-\lambda_{s l} / 2} .
\end{aligned}
$$

The solution with the linear friction law reads:

$$
V(x)=\left\{\begin{array}{rr}
\frac{b_{1} x+b_{2}}{\left(\lambda_{s l} / 2-x\right)^{\frac{1-\epsilon}{2}}\left(\lambda_{s l} / 2+x\right)^{\frac{1+\epsilon}{2}}}, & |x|<\lambda_{s l} / 2 \\
0, & |x|>\lambda_{s l} / 2
\end{array}\right.
$$

where

$$
\begin{aligned}
& \tan \frac{\pi \epsilon}{2}=\frac{\varpi c}{2 S}, \quad b_{1}=\frac{\varpi \tau^{*} \cos \frac{\pi \epsilon}{2}}{2 \mu} \\
& b_{2}=\frac{\epsilon \varpi \tau^{*} \lambda_{s l} \cos \frac{\pi \epsilon}{2}}{4 \mu}+\left(u_{2}-u_{1}\right) \frac{\cos \frac{\pi \epsilon}{2}}{\pi}
\end{aligned}
$$

Extracting the coefficients in front of singularities, one finds the energy flows:

$$
J_{1,2}=\frac{c\left(2 \mu\left(u_{2}-u_{1}\right)+\frac{\pi \varpi \tau^{*} \lambda_{s l}(\epsilon \pm 1)}{2}\right)^{2}}{4 \pi^{2} \varpi \mu \lambda_{s l}} \frac{\sin (\pi \epsilon)}{\epsilon}\left(\frac{a}{\lambda_{s l}}\right)^{ \pm \epsilon} .
$$

The energy flows could have been recovered from expressions (16) for periodic stick-slip in the limit $\alpha \rightarrow 0$, $2 \alpha / k=\lambda_{s l}=$ const.

\section{B.2 Single stick of length $\lambda_{s t} \ll H$}

The solution with finite friction reads:

$$
V(x)=\left\{\begin{array}{rr}
\frac{b_{1} x+b_{2}}{\left(x+\lambda_{s t} / 2\right)^{\frac{1-\epsilon}{2}}\left(x-\lambda_{s t} / 2\right)^{\frac{1+\epsilon}{2}}}, & |x|>\lambda_{s t} / 2 \\
0, & |x|<\lambda_{s t} / 2
\end{array}\right.
$$

Here

$$
\begin{aligned}
& \tan \frac{\pi \epsilon}{2}=\frac{\varpi c}{2 S}, \quad b_{1}=V_{0}=\frac{\varpi \tau^{*} \cot \frac{\pi \epsilon}{2}}{2 \mu}, \\
& b_{2}=-\frac{\varpi \epsilon \lambda_{s t} \tau^{*} \cot \frac{\pi \epsilon}{2}}{4 \mu}+\left(\left.u_{x}\right|_{x=+\infty}-\left.u_{x}\right|_{x=-\infty}\right) \frac{\cot \frac{\pi \epsilon}{2}}{\pi} .
\end{aligned}
$$

\section{Small height solutions for almost incompressible media, $1-2 \nu \ll 1$}

The basic idea of the small height approximation is to use the approximate equations of elasticity theory which are valid if the transverse gradients (along the shortest vertical dimension) of displacements are much larger than the longitudinal ones. This is justified by the fact that certain boundary conditions are imposed on the upper and lower surfaces. The approximation is adequate in the regions far from the points of discontinuity in the boundary conditions. In those regions, imposing $u_{y}$ on the boundaries additionally implies that $\partial_{x} u_{y} \ll \partial_{y} u_{x}$. The approximation cannot be used at the distances of the order of $H$ near the crack tips.

Neglecting $\partial_{x}^{2}$ terms in comparison with $\partial_{y}^{2}$ terms in the exact equations, one gets:

$$
\begin{gathered}
12 \partial_{x}\left(\partial_{x} u_{x}+\partial_{y} u_{y}\right)+b_{0}^{2} \partial_{y}^{2} u_{x}=0 \\
12 \partial_{y}\left(\partial_{x} u_{x}+\partial_{y} u_{y}\right)+b_{0}^{2} \partial_{y}^{2} u_{y}=0
\end{gathered}
$$

where $b_{0}^{2}=12(1-2 \nu)$. The case of $b_{0} \sim 1$ was discussed in Sec. 3.2 In the limit $b_{0} \ll 1$ one can conclude from Eq. (45) that $\operatorname{div} u \equiv \partial_{x} u_{x}+\partial_{y} u_{y}$ is independent of $y$. Then, it is easy to see that $u_{x}$ quadratically depends on $y$, and $u_{y}$ is a cubic polynomial in $y$.

\section{C.1 Long stick}

In the stick region, we use stationary boundary conditions to find the solution which is expressed in terms of unknown functions of $x$ :

$$
\begin{aligned}
& \operatorname{div} u=f_{1}(x) \\
& u_{x}=y(y-1) g_{1}(x)+u_{0} y-V_{0} x(y-1) \\
& u_{y}=y(y-1)\left(p_{1}(x)+y q_{1}(x)\right) .
\end{aligned}
$$

Here the lengths are measured in the units of height, and $u_{0}$ is the displacement of the upper boundary with respect to the origin. Together with the first equation of (45), there are four linear differential equations for the 
four functions, which result in $g_{1}^{\prime \prime}-b_{0}^{2} g_{1}=0$. Thus, all functions can be parametrized by two constants:

$$
\begin{aligned}
& g_{1}=a \sinh \left(b_{0} x\right)+b \cosh \left(b_{0} x\right), \quad f_{1}=\frac{1}{2} V_{0}-\frac{1}{6} g_{1}^{\prime}, \\
& q_{1}=-\frac{1}{3} g_{1}^{\prime}, \quad p_{1}=V_{0}-f_{1}=\frac{1}{2} V_{0}+\frac{1}{6} g_{1}^{\prime} .
\end{aligned}
$$

\section{C.2 Long slip}

The same procedure can be used for the solution in the slip region. Here, because of different boundary conditions, $u_{x}$ has another form:

$$
u_{x}=u_{0}+\frac{c}{S} V_{0}(y-1)+(y-1)\left(h_{2}(x)+y g_{2}(x)\right) \text {. }
$$

The presence of the boundary friction (finite $S$ ) makes this problem more difficult. The set of equations is reduced to the third-order differential equation:

$$
4 h_{2}^{\prime \prime}-b_{0}^{2} h_{2}+\frac{c}{S}\left(-h_{2}^{\prime \prime \prime}+b_{0}^{2} h_{2}^{\prime}\right)=0 .
$$

Without friction the solution can be written as

$$
h_{2}=A \sinh \left(b_{0} x / 2\right)+B \cosh \left(b_{0} x / 2\right) .
$$

Small friction changes the balance between the growing and vanishing exponentials on large scales but the final effect can be shown to be negligible.

Functions $g_{2}, p_{2}, q_{2}$ and $f_{2}=\operatorname{div} u$ are expressed in terms of $h_{2}, V_{0}$ and $c / S$, in analogy to Eqs. (49).

\section{C.3 Matching stick and slip solutions}

The solutions found above are good approximations far from the stick-slip boundaries. To match the solutions, one must satisfy global mass and momentum conservations. At every boundary point this will give two relations, which equals the number of unknown constants in every interval.

The condition, that the total horizontal force acting on a piece including the stick-slip boundary is zero, leads to the continuity of divergences: $f_{1}\left( \pm \lambda_{s t} / 2\right)=f_{2}\left(\mp \lambda_{s l} / 2\right)$.

The second condition is the equality of the volume integral of divergence to the surface integral of the displacement. This gives the relations

$$
\pm \frac{1}{4} V_{0} \lambda_{s t}-\frac{1}{6} g_{1}\left( \pm \lambda_{s t} / 2\right)+\frac{2}{3} h_{2}\left(\mp \lambda_{s l} / 2\right)=\frac{1}{2} u_{0} .
$$

Upper and lower signs correspond to the left and right tips of the slip region.

Deriving the conditions, one neglects $1 / \lambda_{s t}$ and $b_{0}$ in comparison with unity. The full set of equations for four constants reads:

$$
\begin{aligned}
& a \sinh \left(b_{0} \lambda_{s t} / 2\right)+4 A \sinh \left(b_{0} \lambda_{s l} / 4\right)=\frac{3 V_{0} \lambda_{s t}}{2} \\
& b \cosh \left(b_{0} \lambda_{s t} / 2\right)-4 B \cosh \left(b_{0} \lambda_{s l} / 4\right)=-3 u_{0}
\end{aligned}
$$

$$
\begin{aligned}
& a \cosh \left(b_{0} \lambda_{s t} / 2\right)-2 A \cosh \left(b_{0} \lambda_{s l} / 4\right)=\frac{3 V_{0}}{b_{0}}, \\
& b \sinh \left(b_{0} \lambda_{s t} / 2\right)+2 B \sinh \left(b_{0} \lambda_{s l} / 4\right)=0 .
\end{aligned}
$$

Energy flow to the rear edge of the slip is given by

$$
\begin{aligned}
J= & \frac{\mu c}{2}\left(\frac{4}{3} B^{2}-\frac{1}{3} b^{2}-u_{0}^{2}-\frac{2 V_{0} a\left(\cosh \left(b_{0} \lambda_{s t} / 2\right)-1\right)}{b_{0}}\right. \\
& \left.-\frac{2 V_{0} b \sinh \left(b_{0} \lambda_{s t} / 2\right)}{b_{0}}+V_{0} u_{0} \lambda_{s t}-\frac{1}{4} V_{0}^{2} \lambda_{s t}^{2}\right) .
\end{aligned}
$$

In the limit of not extremely long stick and slip regions, $H \ll \lambda_{s t}, \lambda_{s l} \ll H / b_{0}$, the expressions for the energy flow are reduced to

$$
J_{1,2}=\frac{\mu c}{2 H}\left(V_{0} \lambda_{s t} \pm u_{0} \frac{2\left(\lambda_{s t}+\lambda_{s l}\right)}{4 \lambda_{s t}+\lambda_{s l}}\right)^{2} .
$$

Relating the displacement $u_{0}$ and the average shear stress $\tau^{*}$ as

$$
u_{0}=\frac{4 \lambda_{s t}+\lambda_{s l}}{4 \lambda_{s t}} \frac{H \tau^{*}}{\mu},
$$

we rewrite the energy flows in the following manner:

$$
J_{1,2}=\frac{\mu c H}{2}\left(\frac{V_{0} \lambda_{s t}}{H} \pm \frac{\tau^{*}}{\mu} \frac{\lambda}{2 \lambda_{s t}}\right)^{2} .
$$

This result differs from the frictionless case of Eqs. (20) only by numerical factors. Moreover, for $\lambda=\lambda_{s t}$ and $V_{0}=$ 0 , the energy conservation, $J=\gamma c$, leads to the result for the critical stress: $\tau^{*}=2 \sigma_{c}$.

\section{References}

1. B. N. J. Persson, Sliding Friction: physical principles and applications (Springer, Heidelberg, 2000).

2. A. Schallamach, Wear 17, 301 (1971).

3. M. Rubio and J. Galeano, Phys. Rev. E 50, 1000 (1994).

4. T. Baumberger, C. Caroli, and O. Ronsin, Phys. Rev. Lett. 88, 075509 (2002).

5. T. Baumberger, C. Caroli, and O. Ronsin, Eur. Phys. J. E 11, 85 (2003).

6. S. M. Rubinstein, G. Cohen, and J. Fineberg, Nature 430, 1005 (2004).

7. E. Gerde and M. Marder, Nature (London), 413, 285 (2001).

8. T. H. Heaton, Phys. Earth Planet. Inter. 64, 1 (1990).

9. H. Yoshizawa, Y. L. Chen, and J. Israelachvili, J. Phys. Chem. 97, 4128 (1993).

10. K. Ranjith and J. R. Rice, J. Mech. Phys. Solids 49, 341 (2001).

11. C. Caroli, Phys. Rev. E 62, 1729 (2000).

12. E. A. Brener, and V. I. Marchenko, JETP Lett. 76, 211 (2002).

13. H. Müller-Krumbhaar, W. Kurz, E. Brener, Solidification in Phase transformations in materials, ed. by G. Kostorz (Wiley-VCH, Weinheim, New York, 2001).

14. I. S. Aranson, L. S. Tsimring, and V. M. Vinokur, Phys. Rev. B 65, 125402 (2002).

15. H. Nakanishi, Phys. Rev. E 61, 3407 (2000).

16. L. B. Freund, J. Geophys. Res. 84, 2199 (1979).

17. E. A. Brener, S. V. Malinin, and V. I. Marchenko (unpublished). 